A novel type of splayed ferromagnetic order observed in $\mathrm{Yb}_{2} \mathrm{Ti}_{2} \mathrm{O}_{7}$

This content has been downloaded from IOPscience. Please scroll down to see the full text.

2016 J. Phys.: Condens. Matter 28426002

(http://iopscience.iop.org/0953-8984/28/42/426002)

View the table of contents for this issue, or go to the journal homepage for more

Download details:

IP Address: 132.166.160.12

This content was downloaded on 30/06/2017 at 09:21

Please note that terms and conditions apply.

You may also be interested in:

Quantum spin ice: a search for gapless quantum spin liquids in pyrochlore magnets

M J P Gingras and P A McClarty

Anisotropic exchange in frustrated pyrochlore $\mathrm{Yb} 2 \mathrm{Ti} 2 \mathrm{O} 7$

H B Cao, A Gukasov, I Mirebeau et al.

Magnetic frustration in the disordered pyrochlore $\mathrm{Yb2GaSbO7}$

$\mathrm{J}$ A Hodges, $\mathrm{P}$ Dalmas de Réotier, A Yaouanc et al.

Single crystal growth, structure and magnetic properties of Pr2Hf2O7 pyrochlore

Monica Ciomaga Hatnean, Romain Sibille, Martin R Lees et al.

Investigations of the effect of nonmagnetic Ca substitution for magnetic Dy on spin-freezing in

Dy2Ti2O7

V K Anand, D A Tennant and B Lake

The crystal and magnetic structures of $\mathrm{LaCa} 2 \mathrm{Fe} 3 \mathrm{O} 8$ and $\mathrm{NdCa} 2 \mathrm{Fe} 3 \mathrm{O} 8$

J M Hudspeth, D J Goossens, A J Studer et al.

Spin reorientation transition in $\mathrm{CaNdFeO} 4$

Shigeaki Oyama, Makoto Wakeshima, Yukio Hinatsu et al.

Bulk magnetization of the heavy rare earth titanate pyrochlores - a series of model frustrated $\underline{\text { magnets }}$

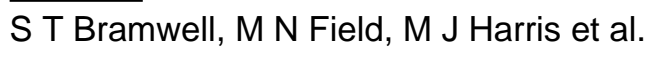




\title{
A novel type of splayed ferromagnetic order observed in $\mathrm{Yb}_{2} \mathrm{Ti}_{2} \mathrm{O}_{7}$
}

\author{
A Yaouanc ${ }^{1,2}$, P Dalmas de Réotier ${ }^{1,2}$, L Keller $^{3}$, B Roessli ${ }^{3}$ and A Forget ${ }^{4}$ \\ ${ }^{1}$ Université Grenoble Alpes, INAC-PHELIQS, F-38000 Grenoble, France \\ 2 CEA, INAC-PHELIQS, F-38000 Grenoble, France \\ ${ }^{3}$ Laboratory for Neutron Scattering and Imaging, Paul Scherrer Institut, 5232 Villigen-PSI, Switzerland \\ 4 SPEC, CEA, CNRS, Université Paris-Saclay, CEA Saclay, 91191 Gif-sur-Yvette Cedex, France \\ E-mail: alain.yaouanc@cea.fr
}

Received 3 February 2016, revised 13 June 2016

Accepted for publication 29 June 2016

Published 25 August 2016

\begin{abstract}
The pyrochlore insulator $\mathrm{Yb}_{2} \mathrm{Ti}_{2} \mathrm{O}_{7}$ has attracted the attention of experimentalists and theoreticians alike for about 15 years. Conflicting neutron diffraction data on the possible existence of magnetic Bragg reflections at low temperature have been published. Here we report the observation of magnetic Bragg reflections by neutron powder diffraction at $60 \mathrm{mK}$. The magnetic diffraction pattern is analyzed using representation theory. We find $\mathrm{Yb}_{2} \mathrm{Ti}_{2} \mathrm{O}_{7}$ to be a splayed ferromagnet as reported for $\mathrm{Yb}_{2} \mathrm{Sn}_{2} \mathrm{O}_{7}$, a sibling compound with also dominating ferromagnetic interactions as inferred from the positive Curie-Weiss temperature. However, the configuration of the magnetic moment components perpendicular to the easy axis is of the all-in-all-out type in $\mathrm{Yb}_{2} \mathrm{Ti}_{2} \mathrm{O}_{7}$ while it is two-in-two-out in the $\mathrm{Yb}_{2} \mathrm{Sn}_{2} \mathrm{O}_{7}$. An overall experimental picture of the magnetic properties emerges.
\end{abstract}

Keywords: magnetic frustration, spin ice, pyrochlore

(Some figures may appear in colour only in the online journal)

\section{Introduction}

The broad interest in geometrically frustrated magnetic materials such as pyrochlore insulator compounds $R_{2} M_{2} \mathrm{O}_{7}(F d \overline{3} m$ space group), where $R$ is a rare earth ion and $M$ a non magnetic element, arises from the large diversity of magnetic ground states and exotic fluctuations and excitations encountered [1-5]. This is due to the combined effects of the geometrical constraint resulting from the topology of the pyrochlore lattice and the action of the different possible interactions. As a consequence, classical and quantum fluctuations are particularly strong. Among the interactions of interest, the crystal field at the $R$ position, the Heisenberg nearest-neighbour exchange interaction as well as its extension beyond the nearest-neighbour $R$ ions $[6,7]$, the anisotropy of the exchange interactions $[8,9]$ and the inevitable dipolar interactions have been considered.

The most remarkable discovery in the realm of geometrically frustrated magnetic materials has been the spin-ice ground state of $\mathrm{Ho}_{2} \mathrm{Ti}_{2} \mathrm{O}_{7}$ in 1997 [10]. It is characterized by dipolar correlations emerging from the topological spin-ice constraint. This translates into pinch-points in the neutron diffuse scattering patterns [11] and magnetic monopole excitations [12-14].

Because of its large ground-state degeneracy, a spin-ice compound does not order magnetically, i.e. it is in a socalled spin liquid state where the spins are strongly correlated although they do not display long range order. Quantum fluctuations are not expected to play a significant role for $\mathrm{Ho}_{2} \mathrm{Ti}_{2} \mathrm{O}_{7}$. However, for some pyrochlore materials strong quantum transverse fluctuations [8,9] could stabilize new magnetic states such as a quantum spin liquid for which new exotic excitations are expected [3, 5, 15-17].

One of the favourite candidates for this exotic ground state is $\mathrm{Yb}_{2} \mathrm{Ti}_{2} \mathrm{O}_{7}$. Indeed, the exchange interactions are highly anisotropic, with a strong ferromagnetic component akin to the Ising exchange of spin ice [18]. The dominating ferromagnetic component is also suggested by the positive CurieWeiss temperature [19]. Remarkably, the Ising character of the exchange interaction is in contrast to the marked planar anisotropy of the $\mathrm{Yb}^{3+}$ ground state Kramers doublet $[19,20]$. The measured entropy provides evidence for a ground state doublet well isolated from the excited doublets [21-23]. 
The strong specific heat anomaly early reported by Blöte and collaborators at $T_{\mathrm{c}} \simeq 0.21 \mathrm{~K}$ for a polycrystalline sample [21] does not necessarily correspond to dipolar magnetic ordering. A hidden, i.e. non-dipolar, order has been invoked $[24,25]$. Alternatively the coherent magnetic order could be so disrupted by crystal defects that any magnetic Bragg reflections would be too broad to be observable [26]. Due to the long-standing controversy as to the presence of magnetic Bragg reflections [22, 27-32], the magnetic nature of the ground state of $\mathrm{Yb}_{2} \mathrm{Ti}_{2} \mathrm{O}_{7}$ at low temperature is still uncertain. Magnetic Bragg reflections are reported only in [27, 31] which describe data measured on the same crystal. The diversity of the specific heat [31,33-35] and muon spin relaxation $(\mu \mathrm{SR})$ results $[22,23,25,36]$ adds to the difficulty in understanding the ground state. In addition to the sample quality issue first noticed in [34], the low temperature required for the observation of neutron magnetic Bragg reflections is an additional issue. This is particularly true for a powder sample as illustrated by the sibling compound $\mathrm{Yb}_{2} \mathrm{Sn}_{2} \mathrm{O}_{7}$ for which only two out of the three powder neutron diffraction studies have reported the existence of magnetic reflections [37-39].

Our previous successful work for $\mathrm{Yb}_{2} \mathrm{Sn}_{2} \mathrm{O}_{7}$ [37] has motivated us to revisit the case of $\mathrm{Yb}_{2} \mathrm{Ti}_{2} \mathrm{O}_{7}$. Here we report the observation of magnetic Bragg reflections at $60 \mathrm{mK}$ by powder neutron diffraction for the titanate and the determination of its magnetic structure. The result of the analysis is based on crystal symmetry and is at variance with the previously proposed collinear or nearly collinear ferromagnetic structure [27, 31].

\section{Experiment}

The syntheses of the present sample and the one studied in [22] were similar. A single phase polycrystalline sample was prepared by heating the constituent oxides up to $1400{ }^{\circ} \mathrm{C}$ with four intermediate grindings. Interestingly, a similar procedure was used for $\mathrm{Nd}_{2} \mathrm{Sn}_{2} \mathrm{O}_{7}$ and gave an excellent sample [40]. A sharp singularity appears in the specific heat at $T_{\mathrm{c}} \approx 255$ $\mathrm{mK}$ for a previously prepared $\mathrm{Yb}_{2} \mathrm{Ti}_{2} \mathrm{O}_{7}$ sample [33]. Similar peaks have always been reported in the literature for powders of $\mathrm{Yb}_{2} \mathrm{Ti}_{2} \mathrm{O}_{7}[21,23,25,26]$.

The neutron diffraction measurements were performed at the cold neutron powder diffractometer DMC of the SINQ facility, Paul Scherrer Institut (PSI), Switzerland. In the evening before the experiment $\approx 10 \mathrm{~g}$ of powder were inserted into a copper container filled at room temperature with 10 bars of helium and sealed. The container was mounted in a liquid helium cryostat. The dilution refrigerator was started the next morning and the neutron scattering experiment was started after stabilization of the temperature at $60 \mathrm{mK}$. The measurement lasted for almost two days at this temperature, followed by two and a half days at $1.5 \mathrm{~K}$ and again at $60 \mathrm{mK}$ for another night. As just explained, two measurements were carried out at $60 \mathrm{mK}$, before and after the $1.5 \mathrm{~K}$ measurement. The appearance of additional intensity at $60 \mathrm{mK}$ was reproducible, for the second run with lower counting statistics, of course, but clearly visible. The two data sets recorded at 60 $\mathrm{mK}$ were finally combined. The magnetic diffraction pattern results from the difference of the data sets at $60 \mathrm{mK}$ and $1.5 \mathrm{~K}$.

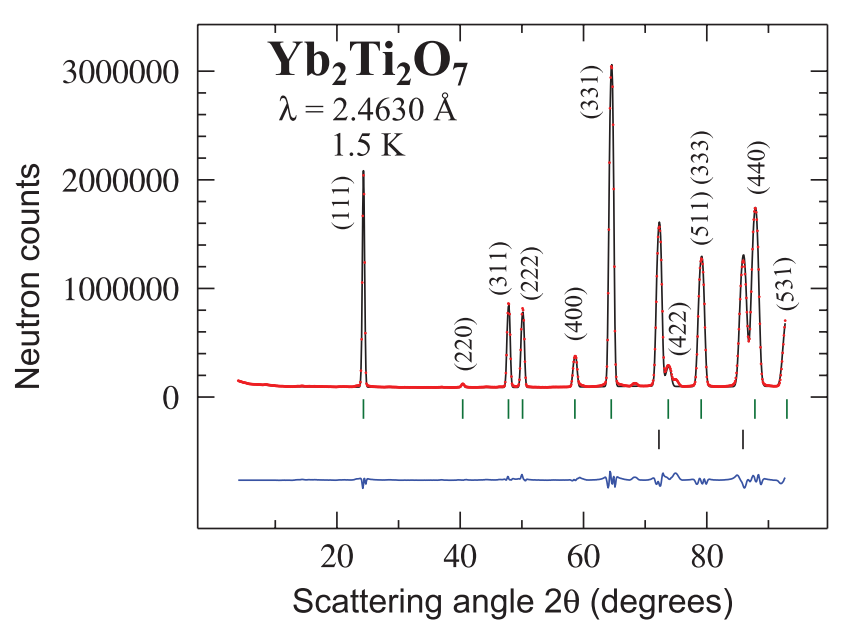

Figure 1. Neutron diffraction pattern for a powder of $\mathrm{Yb}_{2} \mathrm{Ti}_{2} \mathrm{O}_{7}$ at $1.5 \mathrm{~K}$. The intensity is plotted versus the scattering angle $2 \theta$. Neutrons of wavelength $2.4630 \AA$ were used. The solid black line results from a Rietveld refinement [41]. The difference between the experimental data and the refinement is shown at the bottom. The observed reflections are labeled by Miller indices. The vertical markers indicate the positions of the Bragg peaks in the face-centered cubic $F d \overline{3} \mathrm{~m}$ space group. The other two markers correspond to Bragg peaks of the $\mathrm{Cu}$ sample container. We get the lattice parameter of $\mathrm{Yb}_{2} \mathrm{Ti}_{2} \mathrm{O}_{7}, a=10.0220(5) \AA$ and the free parameter for the oxygen position at the $2 \mathrm{~mm}$ site, $x=0.332$ (1).

\section{Crystal structure from neutron diffraction}

The diffraction pattern recorded at $1.5 \mathrm{~K}$ is shown in figure 1 . Our results are consistent with available neutron [35] or synchrotron x-ray diffraction data [42] recognizing that these latter experiments have been performed at room temperature. Separate measurements at 1.9 and $20 \mathrm{~K}$ using a high resolution neutron powder diffractometer were performed ${ }^{5}$. The data confirm the high quality of the powder, with the presence of a ultra-minority rutile-type $\mathrm{TiO}_{2}$ phase amounting to $0.9 \%$ wt. Its signature is visible at $2 \theta \approx 68^{\circ}$ in figure 1 . Concerning the pyrochlore phase, the partial occupancy of the $\mathrm{Ti}^{4+}$ site by $\mathrm{Yb}^{3+}$ ions - the so-called stuffing already reported for crystals [35] — is found to be 0.002 (1), i.e. fully negligible.

\section{Magnetic structure from neutron diffraction}

The magnetic diffraction pattern is displayed in figure 2(a). Remarkably, magnetic reflections are observed at positions corresponding to the face-centered cubic reciprocal lattice. Therefore a magnetic order is found characterized by a $\mathbf{k}=0$ propagation wavevector. Since the width of the magnetic reflections is resolution limited, the magnetic order is long range. Rietveld refinements of the magnetic diffraction pattern were attempted with FullProf [43] for the magnetic structures allowed by the $F d \overline{3} m$ space group. As for $\mathrm{Yb}_{2} \mathrm{Sn}_{2} \mathrm{O}_{7}[37,38]$ the data were found incompatible with the $F d \overline{3} m$ crystal symmetry. Then we searched for a solution in the tetragonal space group $I 4_{1} /$ amd with the help of the program BasIreps of the FullProf suite [44]. Among the highest non-isomorphic subgroups of $F d \overline{3} m$, this group is the only group simultaneously allowing

${ }^{5}$ A detailed report of this structural study will be published elsewhere. 

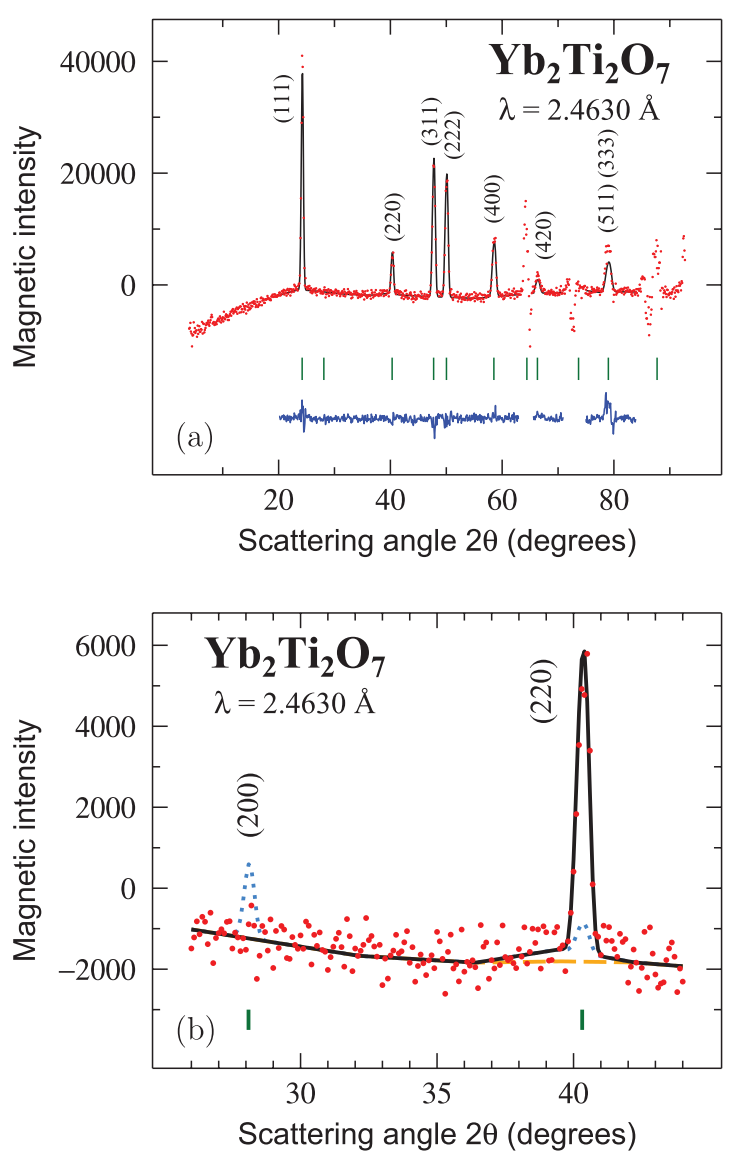

Figure 2. (a) Magnetic neutron scattering pattern given by the difference between $60 \mathrm{mK}$ and $1.5 \mathrm{~K}$ data sets for a powder of $\mathrm{Yb}_{2} \mathrm{Ti}_{2} \mathrm{O}_{7}$ versus the scattering angle $2 \theta$. The experimental data are drawn as red dots. The observed magnetic reflections are labeled by Miller indices. The solid black line results from a Rietveld refinement according to the all-in-all-out splayed ferromagnetic model. The difference between the experimental data and the refinement is shown at the bottom. The vertical markers indicate the positions of the possible Bragg peaks in the $\mathbf{k}=0$ magnetic structure. Data near $2 \theta=64^{\circ}$ and $72^{\circ}$ and below $20^{\circ}$ and above $84^{\circ}$ are not included in the refinement because they are strongly influenced by the sample environment. (b) Details of the data in the 24-46 degrees angular range, showing a comparison of the prediction of the models considered in the main text. The black line is reproduced from panel (a) while the orange dashed line (lightblue dotted) line corresponds to the prediction for the collinear (two-in-two-out splayed) ferromagnetic structure.

ferromagnetic and antiferromagnetic components [45]. In contrast to the case of $\mathrm{Yb}_{2} \mathrm{Sn}_{2} \mathrm{O}_{7}$, no refinement according to this magnetic structure can account for the magnetic pattern of $\mathrm{Yb}_{2} \mathrm{Ti}_{2} \mathrm{O}_{7}$; see the discussion section for more details. In fact, we found the magnetic pattern to be consistent with the magnetic structure described by spontaneous magnetic moment components as given in table 1 . Relative to the stannate, the signs of the $X$ and $Y$ components for sites 1 and 4 are reversed.

Looking for the symmetry elements that keep the magnetic structure invariant, the $I m^{\prime} m^{\prime} a$ group was inferred to be the possible magnetic space group in which $\mathrm{Yb}_{2} \mathrm{Ti}_{2} \mathrm{O}_{7}$ orders ${ }^{6}$.

${ }^{6}$ For an introduction to magnetic space groups, see, e.g. [55] and references therein. Note that the magnetic structure symmetry elements must be consistent with the underlying crystal lattice irrespective of the first order character of the magnetic transition [56].
Table 1. Components of the four spontaneous magnetic moments of the $\mathrm{Yb}^{3+}$ ions in a tetrahedron.

\begin{tabular}{lllllll}
\hline Site & $X$ & $Y$ & $Z$ & $m_{\mathrm{sp}}^{X}$ & $m_{\mathrm{sp}}^{Y}$ & $m_{\mathrm{sp}}^{Z}$ \\
\hline 1 & $\frac{1}{2}$ & $\frac{1}{2}$ & $\frac{1}{2}$ & $0.30(1)$ & $0.30(1)$ & $0.86(1)$ \\
2 & $\frac{1}{2}$ & $\frac{1}{4}$ & $\frac{1}{4}$ & $0.30(1)$ & $-0.30(1)$ & $0.86(1)$ \\
3 & $\frac{1}{4}$ & $\frac{1}{2}$ & $\frac{1}{4}$ & $-0.30(1)$ & $0.30(1)$ & $0.86(1)$ \\
4 & $\frac{1}{4}$ & $\frac{1}{4}$ & $\frac{1}{2}$ & $-0.30(1)$ & $-0.30(1)$ & $0.86(1)$ \\
\hline
\end{tabular}

Note: The site positions $X, Y$ and $Z$ and the moment components $m_{\mathrm{sp}}^{X}, m_{\mathrm{sp}}^{Y}$, $m_{\mathrm{sp}}^{Z}$, given in Bohr magneton units at the temperature of $60 \mathrm{mK}$, are expressed respective to the cubic axes. The uncertainties are of statistical origin.

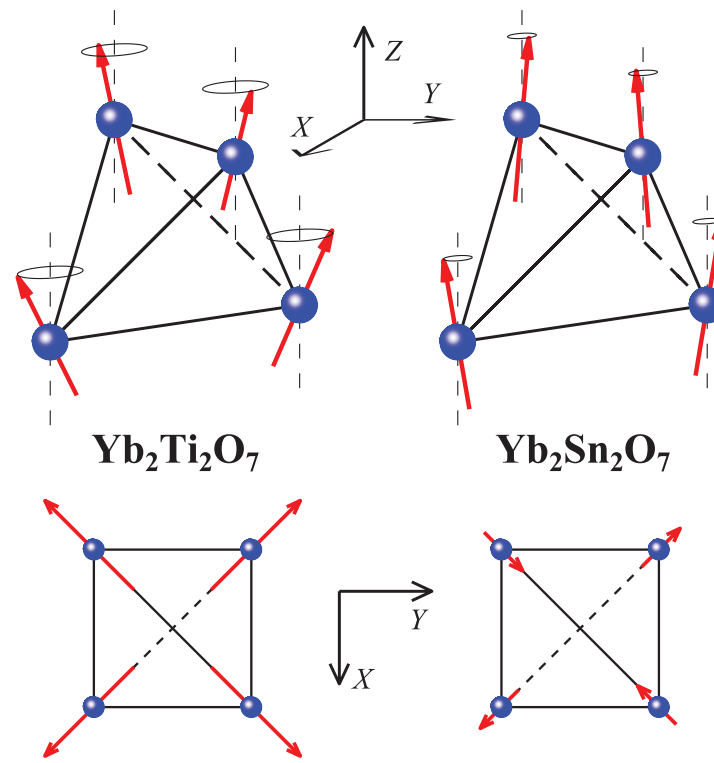

Figure 3. Comparison of the magnetic structures inferred from neutron powder diffraction for $\mathrm{Yb}_{2} \mathrm{Ti}_{2} \mathrm{O}_{7}$ and $\mathrm{Yb}_{2} \mathrm{Sn}_{2} \mathrm{O}_{7}$. Reference frame $(X, Y, Z)$ corresponds to the cubic axes. (Upper panel) Perspective drawing of the magnetic moments (arrows) of the $\mathrm{Yb}^{3+}$ ions (spheres) of a tetrahedron for the two compounds. (Lower panel) Projection of the tetrahedon magnetic moments in the $(X, Y)$ plane. In $\mathrm{Yb}_{2} \mathrm{Ti}_{2} \mathrm{O}_{7}$, while all the projected components point outwards of the represented tetrahedron, in the neighbouring tetrahedra they point inwards. In $\mathrm{Yb}_{2} \mathrm{Sn}_{2} \mathrm{O}_{7}$, for all the tetrahedra, two components are directed inwards and the other two outwards. For these reasons the $\mathrm{Yb}_{2} \mathrm{Ti}_{2} \mathrm{O}_{7}$ and $\mathrm{Yb}_{2} \mathrm{Sn}_{2} \mathrm{O}_{7}$ arrangements of the moment component perpendicular to the ferromagnetic axis are respectively referred to as all-in-all-out and two-in-two-out.

The prime superscript indicates that the symmetry operation is combined with time reversal. The corresponding structural space group is Imma. Interestingly, this latter space group is one of the subgroups of $I 4_{1} /$ amd [46]. The $a$ and $b$ axes of the orthorhombic cell are shorter than the cubic axes by a factor $\sqrt{2}$ and are rotated by $45^{\circ}$ relative to them. A signature of the displacement of the atoms from their position in the cubic space group would be a splitting or a broadening of the Bragg peaks when crossing the magnetic transition. An alternative sensitive signature of such a displacement would be the appearance of nuclear scattering intensity at a position forbidden in the $F d \overline{3} m$ space group but authorised in the Imma space group, e.g. $\left(\begin{array}{lll}0 & 0 & 2\end{array}\right)$. Since neither of the effects is observed, the structural changes are negligible. The $16 \mathrm{~d}$ site of $F d \overline{3} m$ occupied 
Table 2. Comparison of three models for the magnetic structure refinement.

\begin{tabular}{|c|c|c|c|c|c|c|c|}
\hline & \multicolumn{3}{|c|}{ Bragg peaks } & \multirow{2}{*}{$\frac{m_{\mathrm{sp}}^{X}}{\left(\mu_{\mathrm{B}}\right)}$} & \multirow{2}{*}{$\frac{m_{\mathrm{sp}}^{Z}}{\left(\mu_{\mathrm{B}}\right)}$} & \multirow{2}{*}{$\frac{m_{\mathrm{sp}}}{\left(\mu_{\mathrm{B}}\right)}$} & \multirow[t]{2}{*}{$R_{\mathrm{wp}}$} \\
\hline & $\left(\begin{array}{lll}1 & 1 & 1\end{array}\right)$ & $\left(\begin{array}{lll}2 & 0 & 0\end{array}\right)$ & $\left(\begin{array}{lll}2 & 2 & 0\end{array}\right)$ & & & & \\
\hline Collinear FM & 18318 & 0 & 0 & 0 & $0.90(2)$ & $0.90(2)$ & 9.31 \\
\hline Two-in-two-out splayed FM & 22031 & 873 & 410 & $0.14(2)$ & $0.85(1)$ & $0.88(2)$ & 8.91 \\
\hline All-in-all-out splayed FM & 18591 & 0 & 4129 & $0.30(1)$ & $0.86(1)$ & $0.95(2)$ & 5.33 \\
\hline Experiment & $19535(480)$ & $\leqslant 155$ & $4117(152)$ & - & - & - & - \\
\hline
\end{tabular}

Note: The rows give the integrated intensity in arbitrary units at three Bragg positions for the best fit to three models as described in the main text. The last row corresponds to the experimental result. For each of the three models the components for the magnetic moment and its modulus are given. Finally, as a numerical indicator of the fitting agreement, the weighted profile factor [43] $R_{\mathrm{wp}}$ is reported. For reference, its expected value is 4.40.

by the $\mathrm{Yb}^{3+}$ ions transforms and splits into two sites, namely $4 \mathrm{~b}$ and $4 \mathrm{c}$, in Imma. In terms of symmetry the moments at the two sites are allowed to differ. However, within our experimental uncertainties we found them equal, consistent with the ${ }^{170} \mathrm{Yb}$ Mössbauer spectroscopy results [22]. Their amplitude is $m_{\mathrm{sp}}=0.95(2) \mu_{\mathrm{B}}$, a value reasonably close to the estimate $1.1(1) \mu_{\mathrm{B}}$ from neutron diffraction on a single crystal [27], although interpreted with a different magnetic structure-see below_and $1.15 \mu_{\mathrm{B}}$ from Mössbauer spectroscopy [22].

In figure 3 we illustrate the magnetic structures for the two ytterbium compounds. While the projections of the magnetic moments into the $X Y$ plane normal are in an all-in-all-out configuration for the titanate, they adopt a two-in-two-out configuration for the stannate. The angle of a magnetic moment relative to the $Z$ cube edge is $\phi \approx 26^{\circ}$. While $\phi$ is clearly larger than for $\mathrm{Yb}_{2} \mathrm{Sn}_{2} \mathrm{O}_{7}\left(\phi \approx 10^{\circ}\right.$ [37]), $m_{\mathrm{sp}}^{Z}$ is still predominant relative to $m_{\mathrm{sp}}^{\perp}=\sqrt{2} m_{\mathrm{sp}}^{X}$. Hence $\mathrm{Yb}_{2} \mathrm{Ti}_{2} \mathrm{O}_{7}$ belongs to the class of the splayed ferromagnets as its sibling $\mathrm{Yb}_{2} \mathrm{Sn}_{2} \mathrm{O}_{7}$. This is consistent with their positive Curie-Weiss temperature $[19,36]$ and the single-crystal magnetization of $\mathrm{Yb}_{2} \mathrm{Ti}_{2} \mathrm{O}_{7}$ [47]. Although strictly speaking, the three-fold symmetry axis is no longer present in the orthorhombic structure, since the deformation is very small, it is of interest to compute the angle between this direction and the magnetic moment. We find $29(1)^{\circ}$ for sites 1 and 4 and 81 $(1)^{\circ}$ for sites 2 and $3^{7}$. The site numbering refers to table 1 .

\section{Discussion}

The collinear ferromagnetic structure [27] previously deduced from measurements on a single crystal with a specific heat anomaly at $T_{\mathrm{c}} \approx 195 \mathrm{mK}$ [31] is inconsistent with our result. In table 2 we compare the magnetic intensities measured at the position of the first three Bragg peaks authorised in a facecentred cubic structure with the intensities obtained for the best fit to the collinear ferromagnetic structure. For reference we also compare the best fits to the two-in-two-out and to all-in-all-out models. Figure 2(b) graphically illustrates the comparison. The last model is obviously the only one consistent with the data.

\footnotetext{
${ }^{7}$ While, in $\mathrm{Yb}_{2} \mathrm{Sn}_{2} \mathrm{O}_{7}$, a single angle of $65^{\circ}$ between the magnetic moment and the local three-fold axis was deduced from Mössbauer spectroscopy, this angle cannot be directly measured in $\mathrm{Yb}_{2} \mathrm{Ti}_{2} \mathrm{O}_{7}$. This is due to a vanishing quadrupolar interaction in the latter compound [22].
}

As already mentioned, the size of the magnetic moment of the $\mathrm{Yb}^{3+}$ ion inferred from our neutron data is reasonably consistent with the expectation from Mössbauer spectroscopy [22]. The residual difference could be tentatively assigned to short-range magnetic correlations observed in several instances $[31,32,48]$, while the diffraction experiment reported here only picks up the long-range ordered component. The signature of these short-range correlations seems to disappear below $T_{\mathrm{c}}$ [31], but they are still observed in $\mu \mathrm{SR}$ data [48].

A large body of experimental results on $\mathrm{Yb}_{2} \mathrm{Ti}_{2} \mathrm{O}_{7}$ is available in the literature. Because of the mentioned sample quality issues, to get reliable characterizations a selection might be necessary. We propose the cautious criterion of considering only results obtained on polycrystalline samples and on crystals for which magnetic Bragg reflections have been observed. When present in crystals, a sharp peak in the specific heat is observed in the range $0.20-0.25 \mathrm{~K}$ as for polycrystals.

The thermal variation of the entropy deduced from specific heat data is consistent with a well isolated $\mathrm{Yb}^{3+}$ crystal-field Kramers doublet [22, 23]. Apart from the magnetic order described here, measurements on polycrystalline samples have revealed a sharp change in the spin dynamics at $T_{\mathrm{c}}$ in zero field [36]. Signatures of short-range magnetic correlations both in the paramagnetic state through a specific-heat measurement [34] and in the ordered state from $\mu$ SR data $[49,50]$ have been found. Short-range correlations in the ordered state were certainly unexpected. In terms of characteristic fluctuation times, there is a large range of magnetic fluctuation modes, some of them being almost similar in the paramagnetic (still below $\approx 0.7 \mathrm{~K}$ ) and ordered phases [32, 48]. The magnetization

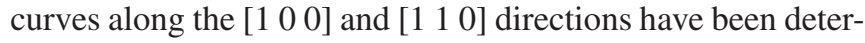
mined [47]. Polarized neutron scattering on crystals shows the presence of a diffuse [ [ $\left.\begin{array}{lll}1 & 1 & 1\end{array}\right]$-rod of scattering and pinchpoint features which develop on cooling [31]. These features are suppressed at $T_{\mathrm{c}}$. The magnetic susceptibility below $T_{\mathrm{c}}$ is stronger in the titanate than in the stannate [48].

A possible theoretical framework for understanding this set of properties could be to consider the paramagnetic phase as the manifestation of a magnetic Coulomb phase with strong quantum fluctuations. It is then tempting to attribute the transition at $T_{\mathrm{c}}$ to the condensation of magnetic monopoles through Higgs's mechanism [31]. However, much work is needed to support this picture. Since spin dynamics is exotic, an inelastic neutron scattering study on reliable crystals is necessary. In particular it should be determined whether a gap exists 
in the excitation spectrum. A scattering technique signature of molecular spin structures such as spin loops involved in the so-called persistent spin dynamics [51] would be of great help. The fact that no spontaneous field has been detected by the $\mu \mathrm{SR}$ technique $[22,23,25]$ is a proof of the existence of pecular spin dynamics related to the Coulomb field [40] and probably the short-range correlations.

On the theoretical side, the ubiquitous slow spin dynamics [22, 36, 47-49], observed even below $T_{\mathrm{c}}$ has to be described. In fact, the most amazing property is not the presence of magnetic order at low temperature-conventional magnetic compounds do display magnetic ordering at low temperature-but the existence of exotic spin correlations in both the paramagnetic and ordered regimes, with the possibility of competing mechanisms at their origin [52].

\section{Conclusions}

$\mathrm{Yb}_{2} \mathrm{Ti}_{2} \mathrm{O}_{7}$ does show magnetic Bragg reflections at low temperature. The structure adopted by its magnetic moments is of the all-in-all-out splayed ferromagnetic type. The actual magnetic moment arrangement-different from the one found for $\mathrm{Yb}_{2} \mathrm{Sn}_{2} \mathrm{O}_{7}$ - should give constraints to the parameters of a Hamiltonian describing the system. While the compound is definitively not a spin liquid system at low temperature, it displays quite exotic spin correlation properties. Although we suspect that they stem from the geometrical constraint imposed by the pyrochlore lattice, this proposal needs to be confirmed by further experimental and theoretical works.

\section{Acknowledgments}

We acknowledge $\mathrm{H}$ Grimmer for an enlighting discussion. This work is based on experiments performed at the Swiss spallation neutron source SINQ, Paul Scherrer Institut, Villigen, Switzerland.

Note added. Two papers on $\mathrm{Yb}_{2} \mathrm{Ti}_{2} \mathrm{O}_{7}$ have recently been published. In one of them, magnetic Bragg reflections have also been observed for a powder sample [53]. The inferred magnetic structure is different from the structure derived in the present report. We note that the limited statistical accuracy of the data reported in [53] makes it difficult to decide between the two models: compare, e.g. the prediction for the intensity at Bragg peak (2 20 ) (table 2) and figure 2(a) of [53]. Concerning the detection reported by Gaudet et al of magnetic intensity above the temperature of the heat capacity peak, we note that the magnetic transition is first order and that the magnetic and paramagnetic phases were found to coexist over a sizable temperature interval [22]. We suggest this is an explanation for the persistence of a magnetic signal above $\approx 0.26 \mathrm{~K}$ in [53]. The work by Gaudet et al also shows that the magnetic excitation spectrum is a gapless continuum. The same group has also reported on crystalline electric field (CEF) measurements and analysis for a stoichiometric and a stuffed sample [54]. The CEF level scheme is similar in both cases and agrees with the previously published scheme [20].

\section{References}

[1] Moessner R and Ramirez A P 2006 Phys. Today 59 24-9

[2] Gardner J S, Gingras M J P and Greedan J E 2010 Rev. Mod. Phys. 8253

[3] Balents L 2010 Nature 464199

[4] Lacroix C, Mendels P and Mila F (ed) 2011 Introduction to Frustrated Magnetism (Springer Series in Solid-State Sciences vol 164) (Berlin: Springer)

[5] Gingras M J P and McClarty P A 2014 Rep. Prog. Phys. 77056501

[6] Wills A S, Zhitomirsky M E, Canals B, Sanchez J P, Bonville P, Dalmas de Réotier P and Yaouanc A 2006 J. Phys.: Condens. Matter 18 L37-42

[7] Yavors'kii T, Fennell T, Gingras M J P and Bramwell S T 2008 Phys. Rev. Lett. 101037204

[8] Curnoe S H 2007 Phys. Rev. B 75212404

[9] Curnoe S H 2008 Phys. Rev. B 78094418

[10] Harris M J, Bramwell S T, McMorrow D F, Zeiske T and Godfrey K W 1997 Phys. Rev. Lett. 79 2554-7

[11] Fennell T, Deen P P, Wildes A R, Schmalzl K, Prabhakaran D, Boothroyd A T, Aldus R J, McMorrow D F and Bramwell S T 2009 Science 326415

[12] Ryzhkin I A 2005 J. Exp. Theor. Phys. 101481

[13] Castelnovo C, Moessner R and Sondhi S L 2008 Nature 45142

[14] Morris D J P et al 2009 Science 326411

[15] Onoda S and Tanaka Y 2010 Phys. Rev. Lett. 105047201

[16] Savary L and Balents L 2012 Phys. Rev. Lett. 108037202

[17] Shannon N, Sikora O, Pollmann F, Penc K and Fulde P 2012 Phys. Rev. Lett. 108067204

[18] Ross K A, Savary L, Gaulin B D and Balents L 2011 Phys. Rev. X 1021002

[19] Hodges J A, Bonville P, Forget A, Rams M, Królas K and Dhalenne G 2001 J. Phys.: Condens. Matter 13 9301-10

[20] Bertin A, Chapuis Y, Dalmas de Réotier P and Yaouanc A 2012 J. Phys.: Condens. Matter 24256003

[21] Blöte H W J, Wielinga R F and Huiskamp W J 1969 Physica 43549

[22] Hodges J A et al 2002 Phys. Rev. Lett. 88077204

[23] Chang L J, Lees M R, Watanabe I, Hillier A D, Yasui Y and Onoda S 2014 Phys. Rev. B 89184416

[24] Thompson J D, McClarty P A, Rønnow H M, Regnault L P, Sorge A and Gingras M J P 2011 Phys. Rev. Lett. 106187202

[25] D’Ortenzio R M et al 2013 Phys. Rev. B 88134428

[26] Ross K A et al 2011 Phys. Rev. B 84174442

[27] Yasui Y et al 2003 J. Phys. Soc. Japan 72 3014-5

[28] Bonville P, Hodges J A, Bertin E, Bouchaud J P, Dalmas de Réotier P, Regnault L P, Rønnow H M, Sanchez J P, Sosin S and Yaouanc A 2004 Hyperfine Interactions 156-7 103-11

[29] Gardner J S, Ehlers G, Rosov N, Erwin R W and Petrovic C 2004 Phys. Rev. B 70180404

[30] Ross K A, Ruff J P C, Adams C P, Gardner J S, Dabkowska H A, Qiu Y, Copley J R D and Gaulin B D 2009 Phys. Rev. Lett. 103227202

[31] Chang L J, Onoda S, Su Y, Kao Y J, Tsuei K D, Yasui Y, Kakurai K and Lees M R 2012 Nat. Commun. 3992

[32] Robert J, Lhotel E, Remenyi G, Sahling S, Mirebeau I, Decorse C, Canals B and Petit S 2015 Phys. Rev. B 92064425

[33] Dalmas de Réotier P, Glazkov V, Marin C, Yaouanc A, Gubbens P C M, Sakarya S, Bonville P, Amato A, Baines C and King P J C 2006 Physica B 374-5 145-7

[34] Yaouanc A, Dalmas de Réotier P, Marin C and Glazkov V 2011 Phys. Rev. B 84172408 
[35] Ross K A, Proffen T, Dabkowska H A, Quilliam J A, Yaraskavitch L R, Kycia J B and Gaulin B D 2012 Phys. Rev. B 86174424

[36] Yaouanc A, Dalmas de Réotier P, Bonville P, Hodges J A, Gubbens P C M, Kaiser C T and Sakarya S 2003 Physica B 326 456-9

[37] Yaouanc A et al 2013 Phys. Rev. Lett. 110127207

[38] Lago J, Živković I, Piatek J O, Álvarez P, Hüvonen D, Pratt F L, Dí az M and Rojo T 2014 Phys. Rev. B 89024421

[39] Dun Z L, Choi E S, Zhou H D, Hallas A M, Silverstein H J, Qiu Y, Copley J R D, Gardner J S and Wiebe C R 2013 Phys. Rev. B 87134408

[40] Bertin A et al 2015 Phys. Rev. B 92144423

[41] Rietveld H M 1969 J. Appl. Crystallogr. 2 65-71

[42] Baroudi K, Gaulin B D, Lapidus S H, Gaudet J and Cava R J 2015 Phys. Rev. B 92024110

[43] Rodriguez-Carvajal J 1993 Physica B 19255

[44] www.ill.eu/sites/fullprof

[45] Mirebeau I, Apetrei A, Rodríguez-Carvajal J, Bonville P, Forget A, Colson D, Glazkov V, Sanchez J P, Isnard O and Suard E 2005 Phys. Rev. Lett. 94246402

[46] Hahn T (ed) 2005 International Tables for Crytallography 5th edn (Dordrecht: Springer)
[47] Lhotel E, Giblin S R, Lees M R, Balakrishnan G, Chang L J and Yasui Y 2014 Phys. Rev. B 89224419

[48] Maisuradze A, Dalmas de Réotier P, Yaouanc A, Forget A, Baines C and King P J C 2015 Phys. Rev. B 92094424

[49] Yaouanc A, Maisuradze A and Dalmas de Réotier P 2013 Phys. Rev. B 87134405

[50] Dalmas de Réotier P, Yaouanc A and Maisuradze A 2014 J. Phys.: Conf. Ser. 551012005

[51] Yaouanc A, Dalmas de Réotier P, Bertin A, Marin C, Lhotel E, Amato A and Baines C 2015 Phys. Rev. B 91104427

[52] Jaubert L D C, Benton O, Rau J G, Oitmaa J, Singh R R P, Shannon N and Gingras M J P 2015 Phys. Rev. Lett. 115267208

[53] Gaudet J, Ross K A, Kermarrec E, Butch N P, Ehlers G, Dabkowska H A and Gaulin B D 2016 Phys. Rev. B 93064406

[54] Gaudet J, Maharaj D D, Sala G, Kermarrec E, Ross K A, Dabkowska H A, Kolesnikov A I, Granroth G E and Gaulin B D 2015 Phys. Rev. B 92134420

[55] Rodríguez-Carvajal J and Bourée F 2012 EPJ Web Conf. 2200010

[56] Bertaut E F 1981 J. Magn. Magn. Mater. 24267 